\title{
A modelling framework for assessing the impact of green mobility technologies on energy systems
}

\author{
Jonas Schnidrig ${ }^{a, C A}$, Tuong-Van Nguyen ${ }^{a, b}$, Xiang Li $^{a}$ and François Maréchal ${ }^{a}$ \\ a Industrial Process and Energy Systems Engineering Group (IPESE), École Polytechnique Fédérale de Lausanne \\ (EPFL), 1950 Sion, Switzerland, jonas.schnidrig@epfl.ch (CA) \\ ${ }^{b}$ Section of Thermal Energy, Technical University of Denmark, 2800 Kongens Lyngby, Denmark
}

\begin{abstract}
:
A successful decarbonisation of the European Union, coupled with a high integration of renewable energy and ambitious targets for energy efficiency, can only be reached with a significant contribution from the transportation sector. It currently represents a quarter of the total greenhouse gas emissions and is shifting from fossil fuels to alternative energy carriers (biofuels, e-hydrogen, electricity) and propulsion systems (hybrid, electric and fuel-cell vehicles). Decarbonising this sector can follow multiple pathways, each having different costs, impacts and implications for the other sectors (industry, residential and services). This paper presents a method to analyse the impact of each decarbonisation pathway in the mobility sector on the overall energy system, using the EnergyScope model. The proposed methods include: (i) an estimation of the hourly demand profiles for short- (local) and long-distance mobility, using annual projections and traffic measurements; (ii) the development of black-box vehicle models of road, rail and aviation technologies; (iii) the modelling of the associated infrastructures, from the fuel conversion processes to the charging stations; and (iv) the use of Monte-Carlobased tools to account for technical and economic uncertainties. This method allows to assess the effects of mobility decarbonisation pathways on the energy system, from the large-scale deployment of vehicle-to-grid technologies to the integration of biofuel- and hydrogen-based vehicles. France has been taken as case study, considering 2050 as time horizon. The results showed the importance of a holistic approach to suggest costand energy-efficient decarbonisation pathways in the transport sector that can affect the overall energy system.
\end{abstract}

\section{Keywords:}

Energy system; Mobility; Decarbonisation

\section{Introduction}

The primary world energy consumption has been more than doubled in the last forty years (factor 2.4) and is covered at more than $80 \%$ by fossil fuels [1]. The same trend can be observed in Europe, with $74 \%$ of the primary energy consumption from fossil fuels, 10\% from nuclear energy and 15\% from renewable sources in 2018. These increases can largely be attributed to the global economic growth, with the largest rise in primary and final energy-demand coming from non-OECD countries. This development is expected to build on the use of fossil fuels, the driving force behind the OECD countries' development in the past [2]. At the European level, this dynamic is different, as population and economic growth grows moderately, which goes in parallel to the energy consumption.

The European Union has drawn up an "Energy Roadmap 2050", within the "European Green Deal" in 2019, which has the goal of a greenhouse gas emission free economy by 2050. This transition requires a shift from fossil fuels to renewable energies and a reduction in the final energy consumption in all sectors (energy, industry, residential, services and mobility) [3].

In the last years, the transportation sector has accounted for about a third of the final energy consumption and a quarter of the total greenhouse gas emissions of the European Union. Private mobility relies heavily, at about $90 \%$, on the use of petroleum fuels (diesel and gasoline) [4]. The ecological awareness of the population is reflected in the change of composition of the new vehicles fleet. Compared to 2018, 22.9\% more electric vehicles, $159.8 \%$ more hybrids and $33.6 \%$ alternative drives were sold in 2019 . The emissions of electric vehicles, trains, and alternative vehicles, are correlated to the energy mix of the electric grid, which varies strongly from one country to another. 
Energy system modelling can focus either on a specific sector or take into account all sectors. The impact of the end-use energy demands in different sectors on the design and operation of our energy systems should be assessed by modelling the whole energy system. A greater penetration of electric vehicles is likely to occur in parallel with an intensified electrification of the industrial, service and residential sectors, for example with the integration of heat pumps for heating. These shifts will result in greater electricity consumption, with a possible competition between the different sectors, large deployment of renewable energies and integration of storage technologies.

Several energy models have been developed to analyse the impacts of possible energy transition pathways and to guide decision-makers and stakeholders. A review of the existing large-scale models is presented in Limpens et al. [5] (Table A1 1), with the new mobility models added in different time resolutions for comparison.

Green mobility technologies are fairly recent and the impact of a large deployment has not been investigated in details. Biresselioglu et al. [6] identified the driving forces and barriers pushing the evolution towards green mobility, while Shafiei et al. [7] focused on the correlation between taxes and electric vehicles' (EV) penetration for 2010-2017. The energy use associated with electric vehicles was modelled by Nematchoua [8] for the city of Liege (BE), London (GB) and Chicago (USA) by Mohammadi et al. [9]. Their focus was on the correlation between socio-economic parameters and spatial energy consumption variation due to mobility.

Bracco [10] developed a model to analyse the interactions between vehicle-to-grid (V2G) technologies and intermittent renewable sources and assess the variations of the electricity and heat consumption. Calise et al. [11] investigated the possibility of combining photovoltaics and batteries to charge the electric vehicles in the cities of Naples and Salerno (IT). Laurischkat and Jandt [12] conducted a thermo-economic optimisation of sustainable mobility, photovoltaic systems and battery storage, while Muratori et al [13] stressed the importance of a multi-sectoral approach to the energy system when analysing the effect of vehicle fleet changes, as the energetic dependencies of the heating system, electricity demand and mobility are related, due to similar primary energy consumption for example.

The aim of this paper is to investigate various technological options for moving towards green mobility and their impact on the whole energy system with the inter-sectoral connections. These alternatives, together with their efficiencies, costs and impacts are implemented in the Mixed Integer Linear Programming (MILP) model EnergyScope, initially developed with a monthly resolution [14, 15]. It has been further developed with a hourly resolution [5] and to include $\mathrm{CO}_{2}$ flow balances [16].

Different scenarii for future mobility are derived, simulated and optimised, considering their benefits in terms of renewable energy share and greenhouse gas reduction under uncertainty based on the reference scenario. This methodology allows to model the hourly and annual mobility demands based on socio-economic parameters in 2050, assess the economic, environmental and system impacts on the energy system due to the renewable mobility integration and identify the parameters subject to the highest uncertainty. The effect of the electrification of the transport sector and the corresponding electricity demand are assessed, and the stabilisation and relive of the electricity system by using V2G technologies or different charging strategies are analysed.

\section{Methods}

Figure 1 summarises the methodological steps. The pre-calculation allows to determine the model's demands and times series input, by (i) selecting the technologies and scenario-dependent vehicles and infrastructure parameters, (ii) specifying additional constraints per case study and vehicle scenario, allowing (iii) to run the model and store the output variables for Pareto generation or sensitivity analysis. These variables are (iv) post-calculated in order to convert into figures, results and key performance indicators.

\subsection{Modelling framework}

Modelling electric vehicles and the impact of their charging/discharging behaviours on the energy system requires a hourly basis to correctly estimate the size and operation of vehicles and storage technologies. The framework of EnergyScope TD developed by Limpens et al. [5] was used: the end-use energy demands were

\footnotetext{
${ }^{1}$ additional material can be found https://infoscience.epfl.ch/record/287243? \&ln=en
} 


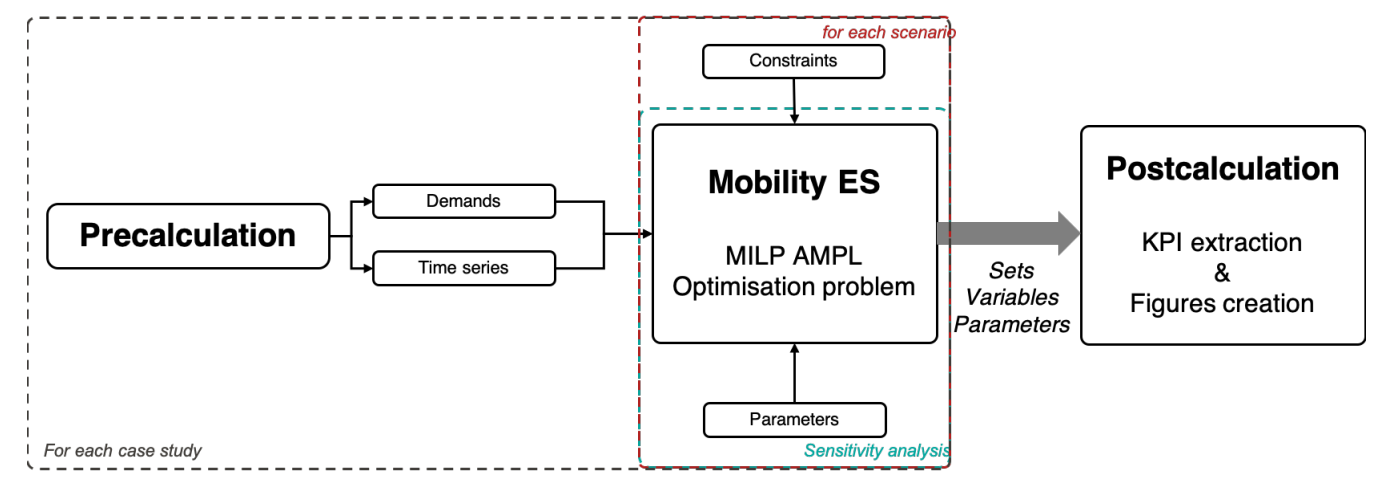

Figure 1: Overview of the computational framework, consisting of (i) pre-calculation with demands and time series definition, (ii) modelling of scenarios and case studies, (iii) computation of Pareto and sensitivity and (iv) the postcalculation.

divided into electricity, heat and mobility, which was on itself split into passenger \& freight mobility. In the present work, passenger mobility was further split into long- and short-distance mobility: these demands are independent from each other, present different time variations and can be associated with different vehicle fleets (short-range electric vehicles can be associated only with short-distance mobility, whilst vehicles running on synthetic fuels can be used for both). Figure A2 shows how to derive the hourly demand profile based on the annual end-uses demands.

EnergyScope figures as energy balance between the hourly demands on one side, and the resources on the other with technologies converting resources to demands under costs and emissions. The modelling framework is depicted in Figure A1, where the red categories correspond to the mobility-related improvements compared to Limpens et al. [5].

Mobility EnergyScope is expressed in MILP, with the key decision variables $\mathbf{F}$ and $\mathbf{F}_{\text {Mult }_{\mathrm{t}}}$, determining the size and the hourly utilisation of the technologies respectively. The model optimises according to two extreme objectives as below and the parametrised Pareto curve.

Economic optimisation The primal objective is the total cost $\mathbf{C}_{\text {tot }}$ (Equation 11) consisting of the sum of the technologies' (tec $\in \mathcal{T E C}$ ) annualised investment $\mathbf{C}_{\mathbf{i n v}}$ and maintenance $\mathbf{C}_{\text {maint }}$ (Equation 3), as of the resources (res $\in \mathcal{R E S}$ ) operation cost $\mathbf{C}_{\mathbf{o p}}$ (Equation 4). The investment cost consists of the multiplication of the specific investment cost $\mathbf{c}_{\mathbf{i n v}}$ with difference of the size parameter $\mathbf{F}$ and the already installed size $\mathrm{f}_{\text {ext }}$ (Equation 2).

$$
\begin{aligned}
\mathbf{C}_{\text {tot }}= & \sum_{t e c} \mathbf{C}_{\mathbf{i n v}}(t e c) \cdot \boldsymbol{\tau}(t e c)+\mathbf{C}_{\text {maint }}(t e c)+\sum_{r e s} \mathbf{C}_{\mathbf{o p}}(r e s) \\
\mathbf{C}_{\mathbf{i n v}}(t e c)= & c_{\text {inv }}(t e c) \cdot\left(\mathbf{F}(t e c)-f_{\text {ext }}(t e c)\right) \\
\mathbf{C}_{\text {maint }}(t e c)= & c_{\text {maint }}(t e c) \cdot \mathbf{F}(t e c) \\
\mathbf{C}_{\mathbf{o p}}(r e s)= & \sum_{h, t d} c_{o p}(r e s) \cdot \mathbf{F}_{\mathbf{t}}(r e s, h, t d) \cdot t_{o p}(h, t d) \\
& \forall \quad r e s \in \mathcal{R E S}, t e c \in \mathcal{T E C}, h \in \mathcal{H}, t d \in \mathcal{T} \mathcal{D}
\end{aligned}
$$

Environmental optimisation The minimisation of the total greenhouse gases emissions is the second objective, measured by the GWP (Global Warming Potential), determined in a similar manner as the total cost where it is split between the emissions caused by construction of technologies $\mathbf{G W P}$ constr and the emissions due to the use of resources $\mathbf{G W P}_{\text {op }}$ (Equations 5.77).

$$
\begin{aligned}
\mathbf{G W P}_{\text {tot }}= & \sum_{i} \mathbf{G W P}_{\mathbf{c o n s t r}}(i)+\sum_{j} \mathbf{G W P}_{\mathbf{o p}}(j) \\
\mathbf{G W P}_{\mathbf{c o n s t r}}(i)= & g w p_{\text {constr }}(i) \cdot \mathbf{F}(i) \\
\mathbf{G W P}_{\mathbf{o p}}(j)= & \sum_{h, t d} g w p_{o p}(j) \cdot \mathbf{F}_{\mathbf{t}}(j, h, t d) \cdot t_{o p}(h, t d) \\
& \forall \quad r e s \in \mathcal{R E S}, t e c \in \mathcal{T E C}, h \in \mathcal{H}, t d \in \mathcal{T} \mathcal{D}
\end{aligned}
$$




\subsection{Mobility demands}

\subsubsection{Hourly demand}

The mobility demand is expressed in [pkm] for passenger transport and [tkm] for freight transport, and is usually given on an annual basis in European statistics. It can be split into long- and short-distance mobility, using a threshold value of $80 \mathrm{~km}$ according to RTE [17]. As for heating systems, which are designed knowing thermal profile demands and possible extreme days in terms of temperature and irradiation, it is essential to derive hourly variations of the mobility demand for sizing adequately the mobility infrastructure and predict peak demands. This is approached, in the present work, by using hourly vehicle counting measurements and deriving mobility profiles. A number of 1513 vehicle counting measurement points in Germany for long distance (BAST [18]) and of 367 in France for short distance mobility (Opendata Paris [19]) were used - depending on the street type and geographical localisation.

\subsubsection{Demand prediction}

Energy system modelling for future years involves the estimation of demands, in particular mobility demand, which has been estimated by regressing the historical demand according to three OECD economic outlook [2] parameters (GDP, number of households, population) in order to determine the weight of the different socioeconomic parameters. The parameters' weights have been determined by minimising the difference of the historic mobility demand $f_{t}^{M o b}$ and the correlated weighted mobility demand $f_{t}^{M o b^{*}}$

$$
\min \sum_{t=1990}^{2019}\left|f_{t}^{M o b^{*}}-f_{t}^{M o b}\right| \quad, \quad f_{t}^{M o b^{*}}=\sum_{n} w_{n} \cdot f_{n, t}
$$

where $\sum_{n} w_{n}=1$ are the weights and $f_{n, t}$ the data of the OECD socio-economic parameter $n$ at year $t$.

\subsection{Mobility technologies}

\subsubsection{Vehicles}

Vehicles are considered as goods (freight) or people (private and public) transporting units over an annual distance by consuming resources. The vehicle specific parameters have been characterised by assuming that people's behaviour in terms of mobility will not change significantly by 2050 . The annually travelled distance $d_{\text {annual }}$ and the load per vehicle $n_{l p v}$ were assumed constant. This allowed to determine the specific energy demand $e^{\text {vehicle }}$ of a given vehicle (private, public and freight mobility) to generate one useful unit of mobility, expressed in pkm for passenger and tkm for freight mobility.

$$
e^{\text {vehicle }}\left[\frac{\mathrm{GWh}}{\mathrm{Mpkm}}\right]=E^{\text {vehicle }} \cdot \frac{8760 \cdot c_{p}^{\text {vehicle }}}{n_{\text {load per vehicle }} \cdot d_{\text {annual }}^{\text {vehicle }}}
$$

The evolution of vehicle costs in the future was estimated using learning curves, which illustrate the relationship between greater production volume and maintenance $\&$ investment costs. They are expressed following a power function of the type:

$$
P\left(x_{t}\right)=P\left(x_{0}\right) \cdot\left(\frac{x_{t}}{x_{0}}\right)^{b}
$$

with $P\left(x_{i}\right)$ being the cumulative production of the reference year $x_{0}$ and the target year $x_{t}$. The slope parameter $b<0$ can be estimated using the learning rate LR:

$$
\mathrm{LR}=\left(1-2^{b}\right)
$$

The different vehicle types are at distinct development states: the learning curve studies of Weiss et al. [20] were used for EV, Ruffini et al. [21] for FCV and no learning curve for ICE vehicles due to their technology readiness level according to Zhao et al. [22]. The maintenance and investment costs for private mobility vehicles were estimated as in Table A2.

The global warming potential related to the LCA analysis of the vehicles was estimated using the EcoInvent database, following the LCIA methodology of the IPCC 2017. The vehicles emissions have been split in the propulsion unit, battery and other units such as fuel cells etc. as visible in Appendix Table A3. 


\subsubsection{Infrastructure}

A shift in mobility behaviour and vehicle fleet will induce modifications to the current infrastructure, with possibly more maintenance of railways and roads, deployment of fuel production units and charging stations, etc. It is assumed that the investment costs of the current roads, railways and waterways are depreciated, and that additional investments are required only if an increase of the end-use mobility demand results in a traffic greater than the planned capacity. Similarly, the investment costs of current gasoline and diesel stations were assumed depreciated, and that these stations could already be used for charging of vehicles running on biofuels. These additional costs for rolling grounds were calculated based on marginal cost estimates of historical data [23], [24] represented in Table 1.

Table 1: Marginal costs of the transportation infrastructure (France).

\begin{tabular}{l|c|ccc}
\hline & $\begin{array}{c}c_{\text {marg }}^{\text {road }} \\
{[€ / \mathrm{pkm}]}\end{array}$ & $\begin{array}{c}c_{\text {maint }}^{2017} \\
{[€ / \mathrm{km}]}\end{array}$ & $\begin{array}{c}d_{s}^{2017} \\
{[\mathrm{~km}]}\end{array}$ & $\begin{array}{c}E U D_{\text {mob }}^{2017} \\
{[\mathrm{pkm}]}\end{array}$ \\
\hline Long Distance & $3.25 \times 10^{-3}$ & 58200 & 20662 & $3.7 \times 10^{11}$ \\
Short Distance & $1.11 \times 10^{-1}$ & 58200 & 1082789 & $5.66 \times 10^{11}$ \\
\hline
\end{tabular}

With complementary fuel technology vehicles, fuel processing units are added, following the technology characterisation in EnergyScope by Moret et al. [14]. The efficiencies of the fuel processes are illustrated in Figure A3 with the specific parameters summarised in Table A4 with output the synthetic fuels in Table A5.

\subsection{Modelling of electric vehicles}

The fuelling process of liquid and gaseous fuels as the temporary impact on the energy system can be neglected compared to electric vehicle charging, as the maximum equivalent fuelling speeds corresponds to $17000 \mathrm{~km} / \mathrm{h}$, compared to $150 \mathrm{~kW}$ charging stations for EVs being 20 times slower. While electricity generation technologies have been taken from EnergyScope TD by Limpens et al. [5], the process of charging for each hour $(h \in \mathcal{H})$ of the typical day $(t d \in \mathcal{T} \mathcal{D}$ ) had to be modelled. Two types of EVs have been integrated: V1G and V2G vehicles, where $\mathrm{V} 2 \mathrm{G}$ are able to use the stored electricity for other purposes and discharge it in the grid, while V1G correspond to the standard vehicles where the battery is used for mobility purposes only.

\subsubsection{Electric vehicle storage}

The total electric vehicle storage capacity $\mathbf{F}(b), b \in \mathcal{B}(\mathcal{E} \mathcal{V})$ is assumed to be proportional to the number of electric vehicles in use $\mathbf{F}(v)$, the fleet size $f_{\text {car,circulation }} \cdot P_{C a r}(v)^{2}$ and the specific energy per car $e^{v e h i c l e}(v)$ for all electric vehicle types $v \in \mathcal{E} \mathcal{V}$ (equation 12 ). While the amount of electricity stored in the electric vehicles batteries Sto Out is equal to electricity needed to power the vehicle for V1G cars, the stored electricity can be used for other purposes for $\mathrm{V} 2 \mathrm{G}$ cars (equation 13 ).

$$
\begin{aligned}
& \mathbf{F}(b)=\frac{\mathbf{F}(v) \cdot e^{\text {vehicle }}(v)}{f_{\text {car }, \text { circulation }} \cdot P_{\text {Car }}(v)} \\
& \forall \quad v \in \mathcal{E} \mathcal{V}, b \in \mathcal{B}(\mathcal{E} \mathcal{V}) \\
& \sum_{b} \text { Stoout }\left(b_{1,2},{ }^{\prime} E L E C^{\prime}, h, t d\right)\left\{\begin{array}{l}
=-\sum_{v_{1}} f\left(v_{1},{ }^{\prime} E L E C^{\prime}\right) \cdot \mathbf{F}_{\mathbf{t}}\left(v_{1}, h, t d\right) \\
\geq-\sum_{v_{2}} f\left(v_{2},{ }^{\prime} E L E C^{\prime}\right) \cdot \mathbf{F}_{\mathbf{t}}\left(v_{2}, h, t d\right)
\end{array}\right. \\
& \forall \quad v_{i} \in \mathcal{E} \mathcal{V}, b \in \mathcal{B}(\mathcal{E} \mathcal{V}), h \in \mathcal{H}, t d \in \mathcal{T} \mathcal{D}
\end{aligned}
$$

\subsubsection{Peak demands}

Peak mobility demand results in fuel and electricity loads on the energy system, leading to spiky and volatile loads on the electricity system in absence of load regulation. The peak demand MaxXmob,demand therefore not only depends on the hourly mobility of the $\mathrm{V} 2 \mathrm{G}$ and $\mathrm{V} 1 \mathrm{G}$ vehicles $\left(\mathbf{F}_{\mathbf{t}}\left(v_{1,2}, h, t d\right) v_{1,2} \in \mathcal{E} \mathcal{V}\right)$, but also

\footnotetext{
${ }^{2} f_{\text {car,circulation }}$ is the fraction of vehicles in circulation, which has been taken as $5 \%$, according to the RTE 2018 data [25]
} 


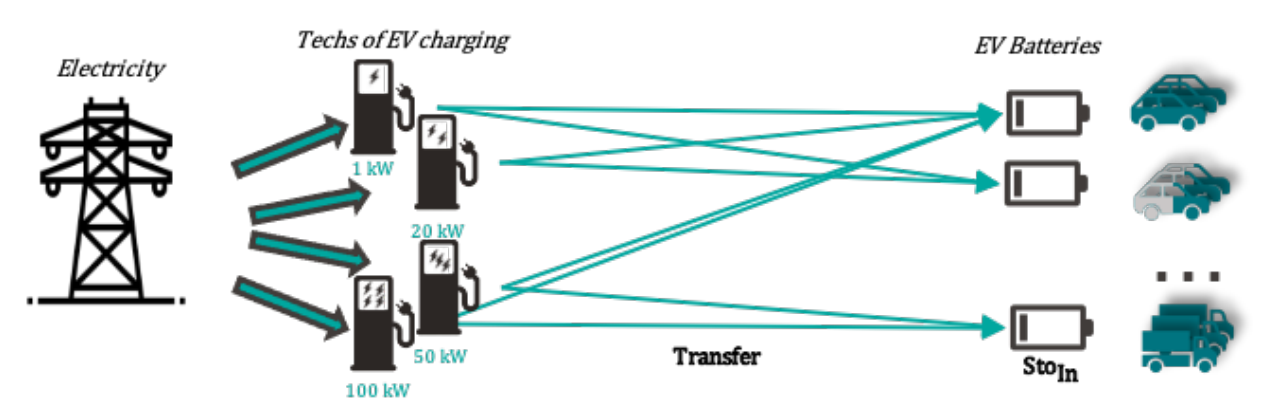

Figure 2: Charging stations modeling illustration with technologies, transfer and levels.

on the number of charges 3 , as for the charging strategy. Peak definition (Equation 14) allows therefore to dimension the electric network, the charging stations, power plants and batteries of the electric vehicles $(b \in$ $\mathcal{B}(\mathcal{E} \mathcal{V})$.

$$
\begin{aligned}
\operatorname{Max}_{\mathbf{X m o b}, \mathbf{d e m a n d}} \geq & \left\{\begin{array}{l}
-\sum_{v} f\left(v_{1,2},{ }^{\prime} E L E C^{\prime}\right) \cdot \mathbf{F}_{\mathbf{t}}\left(v_{1,2}, h, t d\right) \\
-\sum_{v} f\left(v_{1,2},{ }^{\prime} E L E C^{\prime}\right) \cdot \mathbf{F}_{\mathbf{t}}\left(v_{1,2}, h, t d\right)+\sum_{b} \mathbf{S t o}_{\mathbf{i n}}\left(v_{2},{ }^{\prime} E L E C^{\prime}, h, t d\right)
\end{array}\right. \\
& \forall \quad v_{i} \in \mathcal{V}, b \in \mathcal{B}(\mathcal{E V}), h \in \mathcal{H}, t d \in \mathcal{T} \mathcal{D}
\end{aligned}
$$

\subsubsection{Charging stations}

Following the peak demand, electric vehicles are charged (StoIn) at power stations $(c \in \mathcal{C})$. The connection Transfer between the power stations, the vehicle batteries $(b \in \mathcal{B})$ and the grid is summarised in Figure 2. First balance is modelled between electricity exchange of the charging stations and the charging of the batteries (Equations $15 \&$ 16. The additional variable Transfer is created to limit the charging station power type to the different vehicles (Equations 17 \& 18), with the peak power charging output limited in Equations 19 \& 20 .

$$
\begin{aligned}
& \sum_{c} \mathbf{F}_{\mathbf{t}}(c, h, t d) \cdot t_{o p}=\sum_{b} \mathbf{S t o}_{\mathbf{I n}}\left(b,^{\prime} E L E C^{\prime}, h, t d\right) \cdot t_{o p}(h, t d) \\
& \sum_{c} \mathbf{F}(c) \geq \sum_{b} \operatorname{StoIn}_{b}\left(b,^{\prime} E L E C^{\prime}, h, t d\right) \\
& \operatorname{Transfer}(b, c, h, t d) \leq \sum_{j} \mathbf{S t o}_{\mathbf{I n}}\left(b,^{\prime} E L E C^{\prime}, h, t d\right) \cdot t_{o p}(h, t d) \\
& \operatorname{Transfer} \leq c_{a c c e s s, t}(h, t d) \cdot F(c) \\
& \sum_{c} \operatorname{Transfer}(c, b, h, t d)=\mathbf{S t o}_{\mathbf{I n}}\left(b,{ }^{\prime} E L E C^{\prime}, h, t d\right) \\
& \mathbf{F}(c) \geq \sum_{b} \operatorname{Transfer}(c, b, h, t d) \\
& \forall \quad c \in \mathcal{C}, b \in \mathcal{B}, h \in \mathcal{H}, t d \in \mathcal{T} \mathcal{D}
\end{aligned}
$$

\subsubsection{Charging strategies}

The piloting of the charging of electric vehicles batteries $(b \in \mathcal{B}(\mathcal{E} \mathcal{V}))$ allows to flatten the peak demand on the electric grid. Two manual charging strategies have been added, avoiding the charging during hours with a high general electricity demand (Equation 21). The second one aims to avoid trivial charging and discharging, by fixing the charging after the vehicle use $t_{3} ; t_{4}$ in to be at least equal to what was discharged in the previous period $t_{1} ; t_{2}$ (Equation 22).

\footnotetext{
${ }^{3}$ The daily number of charges is assumed to be 1 for a private car and 2-5 for public transport vehicles according to RTE [17].
} 


$$
\begin{aligned}
& \operatorname{Sto}_{\mathbf{I n}}\left(b,^{\prime} E L E C^{\prime}, h^{*}, t d\right)=0 \\
& \sum_{h=t_{3}}^{t_{4}} \operatorname{Sto}_{\mathbf{I n}}\left(i,{ }^{\prime} E L E C^{\prime}, h, t d\right) \geq \sum_{h=t_{1}}^{t_{2}} \operatorname{Sto}_{\text {Out }}\left(b,^{\prime} E L E C^{\prime}, h, t d\right) \\
& \quad \forall \quad b \in \mathcal{B}(\mathcal{E} \mathcal{V}), h \in \mathcal{H}, t d \in \mathcal{T} \mathcal{D}, h_{\text {end }} \geq h^{*} \geq h_{\text {start }} \in \mathcal{H}
\end{aligned}
$$

\subsection{Validation}

The validation of the model has been achieved by comparing the model's output to the energy systems of France in 2017 (Table 2). The major differences between the statistics and the model output can be brought back to (i) efficiencies of the thermal power plants and Diesel vehicles being modelled for future projections with higher efficiencies, (ii) simplified modelling of biomass processes, (iii) lack of data in non-energy, agriculture and aviation purposes on fuel use and carbon emissions and (iv) non-inclusion of power curtailment for photovoltaic

\begin{tabular}{|c|c|c|c|c|c|c|c|c|c|c|c|c|c|c|c|c|}
\hline & \multicolumn{12}{|c|}{ Primary Energy [TWh] } & \multicolumn{4}{|c|}{ Mobility [Mpkm] } \\
\hline & $\overline{\tilde{D}}$ & 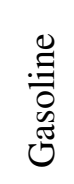 & $\begin{array}{l}\bar{D} \\
\stackrel{\mathscr{D}}{D} \\
\stackrel{0}{0}\end{array}$ & Z & 일 & $\frac{\frac{\tilde{J}}{0}}{\frac{0}{z}}$ & $\begin{array}{l}\vec{a} \\
\dot{\bar{t}} \\
0 \\
0\end{array}$ & 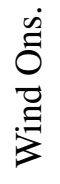 & 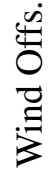 & 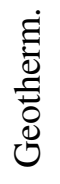 & $\frac{\stackrel{0}{n}}{\pi}$ & $\begin{array}{l}\overline{8} \\
8\end{array}$ & 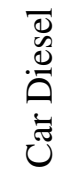 & $\begin{array}{l}\dot{0} \\
\text { Jँ } \\
\dot{\Xi} \\
\dot{\Xi}\end{array}$ & 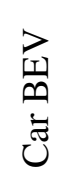 & 胥 \\
\hline Actual 2017 & 114 & 92 & 414 & 446 & 75 & 378 & 9 & 26 & 2 & 0 & 3 & 117 & 520 & 256 & 80 & 311 \\
\hline MILP & 23 & 102 & 450 & 479 & 127 & 386 & 8 & 21 & 2 & 0 & 3 & 115 & 512 & 251 & 79 & 310 \\
\hline$\Delta$ & -91 & 10 & 36 & 33 & 52 & 8 & -1 & -5 & 0 & 0 & 0 & -2 & -8 & -5 & -1 & -1 \\
\hline
\end{tabular}
panels and wind-mills.

Table 2: Model validation: MILP model output vs. actual 2017 values for the France energy system.

\subsection{Sensitivity \& Uncertainty}

\subsubsection{Sensitivity}

A Morris screening method [26] was applied in order to rank the different parameters on the model's objective function value. Despite the drawback of the quantitative nature of the screening, the method allows to classify the parameters in influential and non-influential parameters with a small number of iterations, compared to Sobol methods.

The Morris screening consists of seven steps, where (1) the complete space of possible values of the $k$ input parameters $x$ is discretized in a $p$-levels grid, while (2) taking an initial value set from the $k$ input parameters within the $p$-level grid allowing to calculate (3) the model's objective function value $y$. (4) A new set of parameters is generated varying only one value within its $p$-level, calculating the model's output and repeating these steps $r$-times. (5) The variation of the model output $y$ due to the input $x$ can be determined by the elementary effect (EE) for the $j$-th variable and the $i$-th valuation:

$$
\mathrm{EE}_{j}^{(i)}=\frac{\partial y}{\partial x_{j}} \simeq \frac{y\left(x_{1}, x_{2}, x_{j}+\delta x_{k}\right)-y(\mathbf{X})}{\delta}
$$

(6) The scaling of the elementary effects allows to normalise the inputs parameters variation according to the standard deviation of the models inputs and outputs, allowing (7) to classify the the local derivatives of the solutions compared to the sensitivity of the model according to the indicators (i) $\mu^{*}$, the mean values of the distribution of the standardised absolute elementary effects SEE [27]. The larger $\mu^{*}$ is, the more sensitive the output of the $j$-th input is. $\mu^{*}$ is defined as:

$$
\mu_{j}^{*}=\frac{1}{r} \sum_{i=1}^{r}\left|\mathrm{SEE}_{j}^{(i)}\right|
$$

and (ii) $\sigma$, which is the standard deviation of the distribution of the elementary effects SEE. $\sigma$ measures the nonlinear effects of the $j$ th input on the model output, as well as the effect on the other $k-1$ parameters. $\sigma$ is 
defined as

$$
\sigma_{j}=\sqrt{\frac{1}{r} \sum_{i=1}^{r}\left(\mathrm{SEE}_{j}^{(i)}-\frac{1}{r} \sum_{i=1}^{r} \mathrm{SEE}_{j}^{(i)}\right)^{2}}
$$

Non-influential factors are the group of points being close to the origin (small $\mu^{*}$ and $\sigma$ ). Their effects can be neglected on the models output result in comparison to the other factors, while less-influential factors are values which are located close to the line $\sigma=\mu$. influential non-interacting, are factors below the separation line $\sigma=\mu$. Their influence on the optimisation result is more of additive nature, with influential interacting factors are above the separation line and have a non-linear effect on the result.

\section{Results and discussion}

In order to verify the consistency of the modelling approach, the methodology is applied to the case studies of France in 2050.

\subsection{Mobility estimation}

In a first step, the hourly mobility profile for long and short distance is estimated. Figure 3 identifies the different day type clusters applied to the case of France 2015, with holidays, weekdays and workdays in long distance mobility and missing holidays in short distance mobility, as holidays and weekday cannot be distinguished with the k-means clustering.

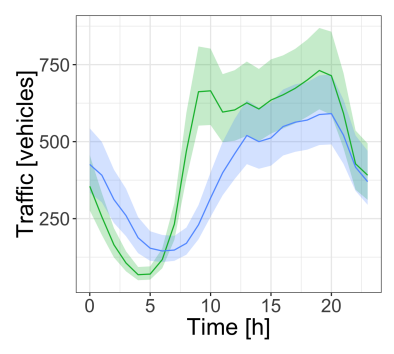

(a) Short Distance

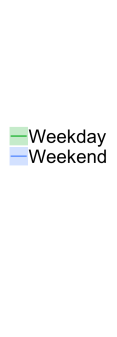

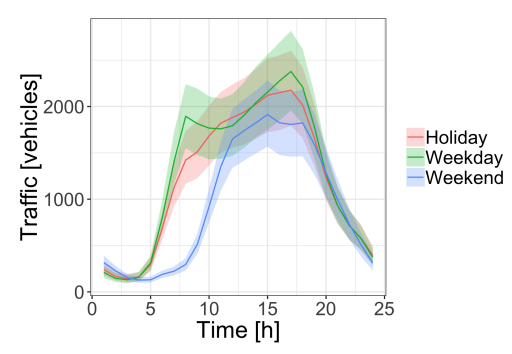

(b) Long Distance

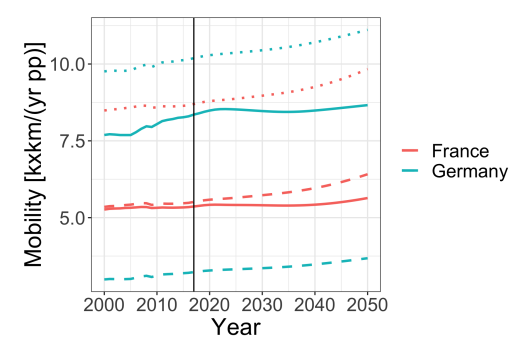

(c) Annual mobility demand evolution

Figure 3: Mobility estimation: (a) \&(b) on the hourly profile estimation with quartiles deviation (transparent), (c) on the Specific annual mobility demand evolution Germany \& France 1990-2050 (dotted SD, dashed, LD, line FR).

In a second step, the annual mobility demand evolution is extrapolated, based on the the OECD parameters where the weights are represented in Table 3. A strong correlation between freight and GDP is visible for both countries, while the population is more influencing the passenger mobility. By extrapolating the mobility demands in 2050 (Figure 3(c)), one can observe a higher specific short distance passenger mobility demand for Germany (11.2\%) while the trend is inverted for long distance mobility $(-93.25 \%)$.

Table 3: Weighting parameters mobility demand extrapolation.

\begin{tabular}{lcccccc}
\hline & \multicolumn{3}{c}{ France } & \multicolumn{3}{c}{ Germany } \\
& SD & LD & Freight & SD & HD & Freight \\
\hline GDP & 0.27 & 0.26 & 0.68 & 0.15 & 0.32 & 0.73 \\
Number of Households & 0.24 & 0.28 & 0.14 & 0.17 & 0.27 & 0.12 \\
Population & 0.54 & 0.46 & 0.18 & 0.68 & 0.41 & 0.15 \\
\hline
\end{tabular}

\subsection{Electric vehicle charging}

The effect of vehicle charging on the energy system can be observed by setting the road fleet to $100 \%$ electric vehicles. Figure 4 displays the charging and discharging of electric road vehicles, with the hourly averaged power for different day types. While on a sunny day in Spring, the renewable resources (PV panels and hydro power) provide enough electricity for the energy system, the EV power curves follow only the mobility demand purposes (Figure 4(a)), V2G and G2V is activated on a cloudy winter day with low renewable potential, allowing to store inter-daily shifted energy production and consumption (Figure 4(b)].

Figure 4(c) corresponds to the subtraction of the mobility-induced power charging and discharging of Figure 
4(b). The differences in use if certain technologies can be explained by (1) the availability of the vehicles during the day, as (2) their capacity to be charged at different charging levels. While PV panels generate electricity at low voltage, wind production is at medium voltage, necessitating $V 2 \mathrm{G}$ vehicles with high-voltage charging capabilities such as trucks or buses. The general trend allows to identify the use of LR vehicles to be charged during midday (people at home or lunch break), while discharged in the early morning or afternoon. Long range BEVs are mainly charged shortly before lunch, to compensate the missing electricity during lunch and the afternoon.

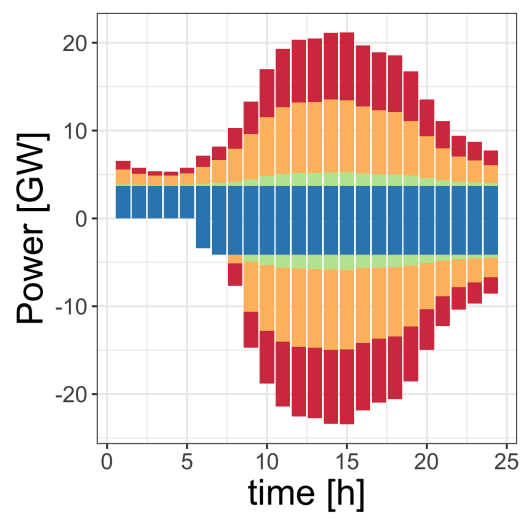

(a) Sunny spring weekend

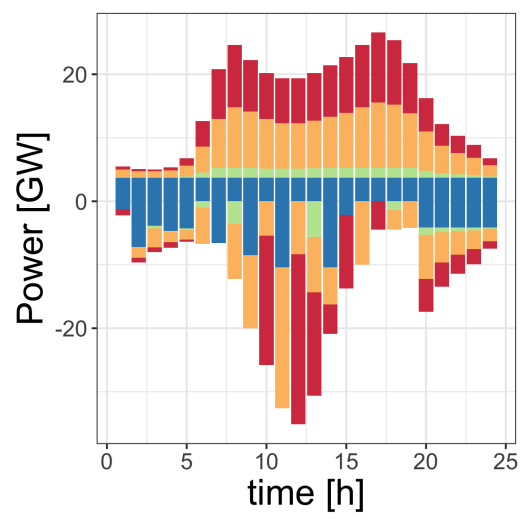

(b) Cloudy winter weekday

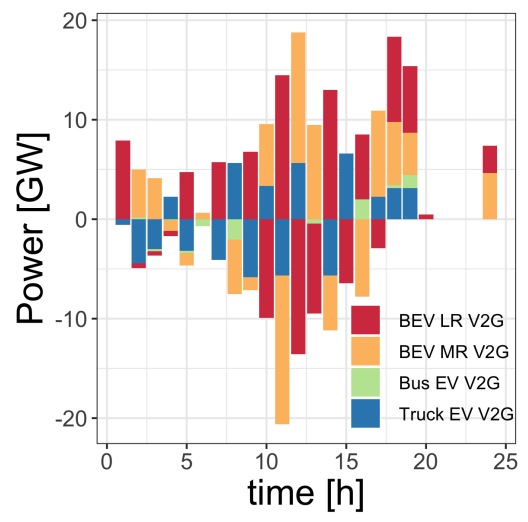

(c) Winter weekday (V2G \& G2V only)

Figure 4: Daily profile of charging (-) and discharging (+) of electric vehicles with $50 \%$ V2G vehicles share for extreme typical days.

\subsection{Decarbonisation pathways}

Different vehicles technologies penetrations allow to assess the economic-ecological optimisation of the technology, visible in Figure 5. BEV correspond to the reference solution in economic and environmental terms (Figure 5(a) and are compensated by fuel cell vehicles (Figure 5(b) when reducing the EV share. Figure 5(c) shows the impact of synthetic and bio fuels on internal combustion engines, where the shares of bio and synthetic fuels are varied. With increasing share, the Pareto front moves backwards, with a bend being visible in high shares, which can be explained by reaching the biomass potential as carbon source, switching towards CCS and DAC technologies, being more expensive but reducing the carbon footprint of the overall system.

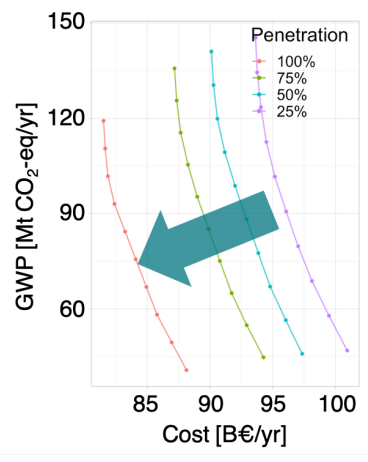

(a) Electric vehicles

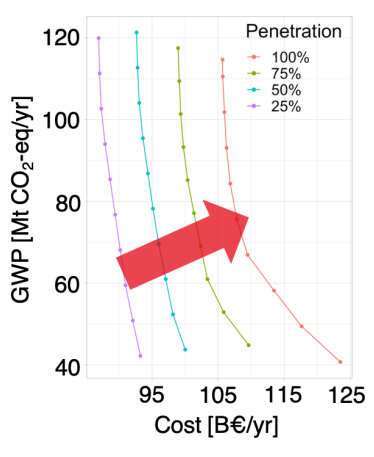

(b) Fuel Cell vehicles

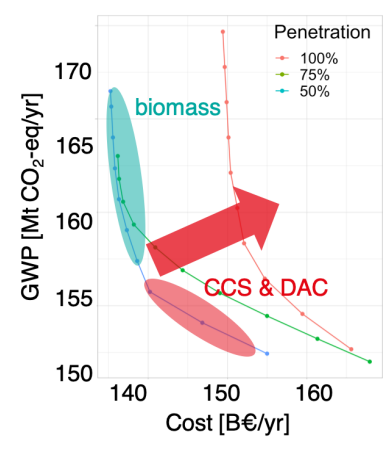

(c) Internal combustion vehicles with biofuels

Figure 5: Pareto curves per vehicle type penetration

The specific primary energy consumption is represented in Figure 6. The reference scenario allows to compare the models' solutions without scenario constraints. France's renewable energy share varies between 27-42\%, as the France's energy policy plans to stick to nuclear power of $61 \mathrm{GW}$ baseline, defining already $58 \%$ of the reference scenarios primary energy consumption.

The lowest consumption that is for all objectives and countries is the scenario without fossil fuel import. The nuclear baseline of France was replaced by more efficient renewable energy technologies generating electricity. 
This results in a primary energy consumption of $10 \mathrm{MWh}$ per year and person for France.

Highest primary energy consumption are the ICE driven scenarios with SF with $40 \%$ higher consumption for the GWP optima and 20\% for the GWP minimisation than the reference scenario. This increase can be brought back to the SF conversion technologies.

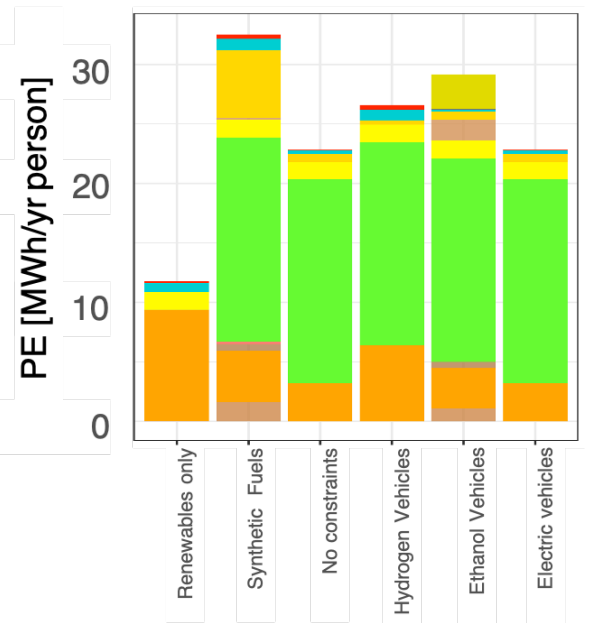

(a) Total Costs

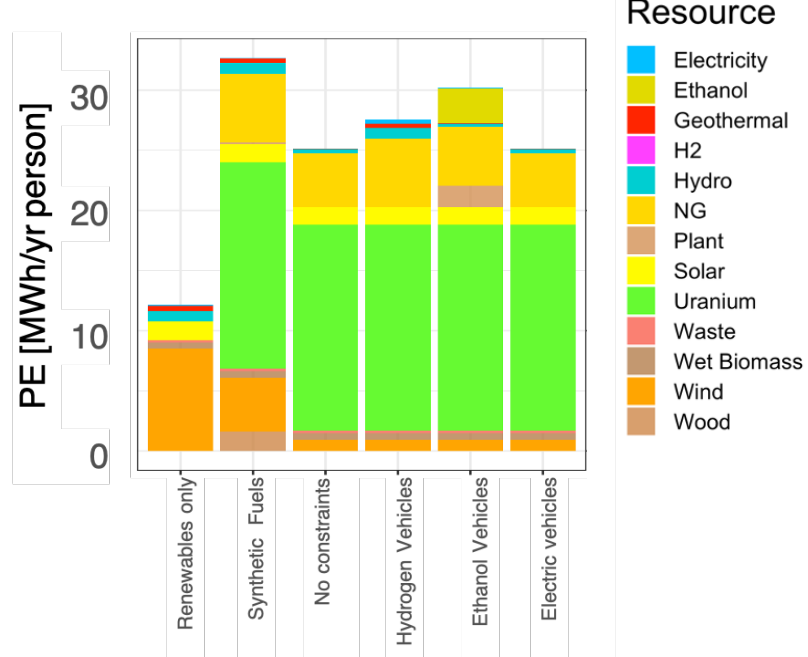

(b) Total GWP

Figure 6: Primary energy consumption per $100 \%$ share scenario by objective function.

\subsection{Sensitivity}

The Morris screening method was proved to be efficient to conduct a preliminary identification of influential and non-influential parameters, as the identification of their nature. The sensitivity analysis has been conducted on both extreme objective functions: total cost and total emissions.

Total Costs Figure 7(a) depicts the 10 most influential parameters of the total costs sensitivity analysis, allowing to classify them according to their influence nature. While influential additive parameters such as the operating cost of Uranium $\left(\mathrm{C}_{\mathrm{op}}^{\text {Uranium }}\right)$ are influencing the total cost objective, the non-linear influential parameters are barely modifying the objective, but inducing modifications on the energy system itself: Photovoltaic panels $\left(\mathrm{C}_{\mathrm{inv}}^{\mathrm{PV}} \& \mathrm{C}_{\text {maint }}^{\mathrm{PV}}\right)$ are in concurrence with Wind $\left(\mathrm{C}_{\mathrm{inv}}^{\mathrm{Wind}}\right)$ and Hydro Dams $\left(\mathrm{C}_{\mathrm{inv}}^{\mathrm{HD}}\right) \&$ Rivers $\left(\mathrm{C}_{\mathrm{inv}}^{\mathrm{HR}}\right)$.

Total GWP In contrary to the total costs sensitivity analysis, all parameters are located in the linear influential zone (Figure 7(b) . While most effect $\left(\mu^{*}>75 \%\right)$ are observed with the household heating and electricity demand $\left(\mathrm{EUD}_{\mathrm{HH}}^{\mathrm{Elec}} \& \mathrm{EUD}_{\mathrm{HH}}^{\mathrm{SH}}\right)$ and the renewable energy potential $\left(\mathrm{f}_{\max }^{\mathrm{Wind}_{\mathrm{off}}} \& \mathrm{f}_{\max }^{\mathrm{PV}}\right)$, all costs and efficiencyrelated parameters have a negligible influence. The absence of emission- \& $\mathrm{CO}_{2}$-related parameters can be explained by the low deviation of those parameters around their base value, as in contrary to the cost evolution, the carbon content of the resources will remain the same.

The absence of mobility-related technologies within the influential parameters underlines the previously shown effect of the electric vehicles as reference scenario in the Pareto fronts. The optimality of electric vehicles in the freight and passenger transport on roads is not as highly sensitive to uncertainty as the renewable power plants, the resources operating costs and the population energy demands.

\section{Discussion}

Each type of vehicle is characterised by a different type of resource, energy use and environmental impact, which results in different trends and impacts on the energy system. 


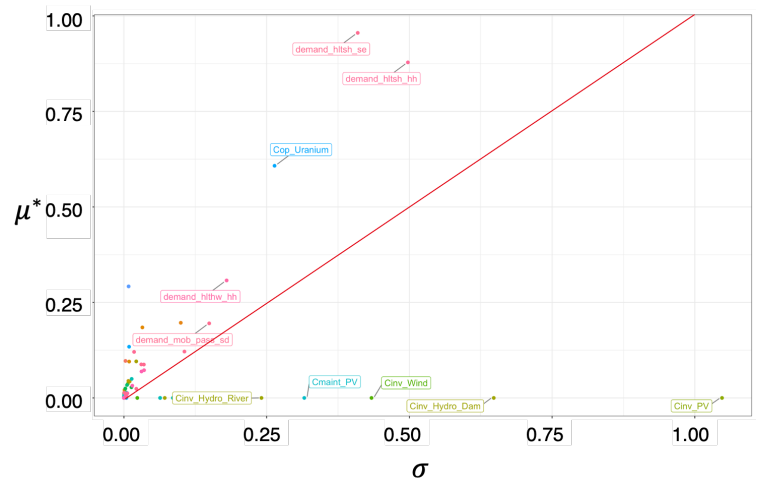

(a) Total Costs

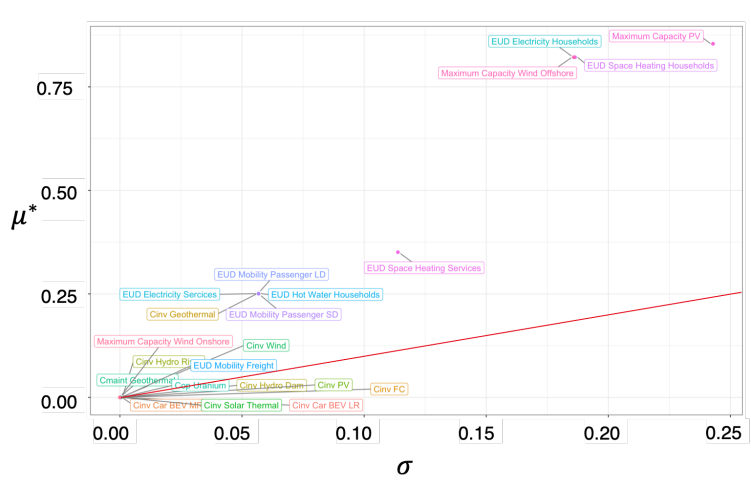

(b) Total GWP

Figure 7: Sensitivity analysis reference scenario France 2050 with Morris screening (zoom in Appendix 2).

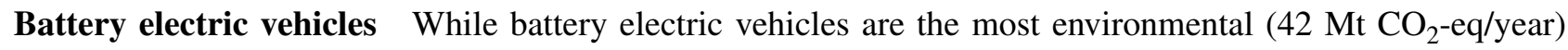
$\&$ economic ( $81 \mathrm{~B} € /$ year) optimal vehicle technology for the whole energy system, its large-scale deployment results in a greater electricity demand, being partially covered by the renewable energy potential $(17-24 \%)$ and completed by possible electricity imports $(1-15 \%)$ or nuclear power $(68-75 \%)$. This enters in competition with the electrification of the heating sector, where heat pumps are being promoted. Smart charging systems and the V2G possibility depends strongly on the proportion of vehicles on the road and allows the temporary storage of electricity from renewable sources, for up to $35 \mathrm{GW}$ peak power and an annual capacity of $1700 \mathrm{GWh}$ for the economic optimum. Smart charging systems can help in levelling down the power peaks ( $-49 \%)$, especially those induced by needs for short-distance mobility.

Synthetic fuels and biofuels While increasing the share in synthetic fuels (diesel and gasoline), the cost and the emissions of the energy system increase by $112 \%$ in system costs and $328 \%$ in emissions compared to electric vehicles. In fact, the limiting parameter is the availability of $\mathrm{CO}_{2}$ to synthesise diesel or gasoline. Hydrogen on the other side can be generated from various sources, either from natural gas through steam methane reforming, or from water and renewable electricity with electrolysis. The amount of carbon required in hydrocarbon-based fuels is limited - it is more economic to recover it from biomass, then from carbon capture on power plants and industrial factories. At high shares of synthetic fuels without fossil fuel import, it becomes necessary to integrate direct air carbon capture technologies, which are still under development.

The production of ethanol and other biofuels is a possibility to continue with the existing vehicle fleet and use ICE. The production of biofuels and the use of ICE are by far less efficient (ecologically and economically) than alternative propulsion systems. Large-scale deployment is also limited because of the lack of carbon sources besides biomass and the power/cement industries, which forces the use of unconventional technologies such as direct air capture. The advantage of these fuels is that the mobility energy vector is independent of the electrical renewable energies and therefore electricity can be used in heat pumps, decarbonising the residential and industrial sectors.

Fuel cell vehicles Despite the initial high investment and maintenance costs, Hydrogen FCV are in competition with BEVs in term of economical optimisation (25\%). By increasing the FCV share, the smaller the electricity demand for BEV gets and the more Hydrogen is generated from electrolysis, allowing to be in concurrence with electric vehicles from an environmental point of view (38\%). At low FCV penetration, the share of $\mathrm{BEV}$ is high, forcing the system to use fossil fuels for heating, inducing the trade-off between electric hydrogen synthesising (electrolysis) and steam methane reforming.

Fuel cell vehicles are the only passenger technology being able to concurrence the battery electric vehicles, while the difference is lowest at long distance mobility, where the vehicle weight starts playing a role.

\section{Conclusion}

In this paper, the modelling framework for the assessment of green mobility in energy systems modelling is presented and applied to the real-world case of two European countries in 2050, by extrapolating based on their socio-economic characteristics and energy policies. 
The adapted approach with higher resolution within the mobility sector allows deeper analyses of the transport system, by quantifying the emission and energy consumption in the transport sector, and in interplay with the other sectors. The integration of green mobility vehicles with their corresponding infrastructure allows to assess the impact of different mobility pathways on the energy system, such as the concurrence between the electrification of sectors, opportunities in system regulation and interplay with renewable energies under the policy induced regulations by states.

The modelling framework allows to integrate further vehicle technologies and to assess their impact on the energy system, as to compare their position to other state of the art vehicles, as for example novel technologies such as carbon capture trucks and hybrid fuel-cell electric vehicles.

Future model development will allow to assess the impact of regional differences within a case study, the exchanges on large and small scale regions, as well as further deepening in precision on the other sectors, such as households, industry and services with their respective interplay.

\section{Acknowledgements}

This project has received funding from the European Union's Horizon 2020 research and innovation program under the Marie Skłodowska-Curie grant agreement No. 754462 - AdvancedGREEN (Towards Advanced fuels and vehicles for GREEN transport).

\section{References}

[1] British Petrol. Statistical Review of World Energy — Energy economics - Home. URL https://www . bp. com/en/global/corporate/energy-economics/statistical-review-of-world-energy. html

[2] OECD stats. Economic Outlook No 103 - July 2018 - Long-term baseline projections. URL https: / / stat s . oecd.org/Index.aspx?DataSetCode=E0103_LTB\#.

[3] European Commission. 2050 long-term strategy. URL https://ec.europa.eu/clima/policies/ strategies/2050_en.

[4] European Union, European Comission, Directorate-General for Mobility and Transport, European Union, and Eurostat. EU Transport in Figures. Publication Office of the European Union. ISBN 978-92-76-03842-9.

[5] Gauthier Limpens, Stefano Moret, Hervé Jeanmart, and Francois Maréchal. EnergyScope TD: A novel open-source model for regional energy systems. 255:113729. ISSN 03062619. doi: 10.1016/j.apenergy.2019.113729. URL https://linkinghub.elsevier.com/retrieve/pii/s0306261919314163.

[6] Mehmet Efe Biresselioglu, Melike Demirbag Kaplan, and Barbara Katharina Yilmaz. Electric mobility in Europe: A comprehensive review of motivators and barriers in decision making processes. 109:1-13. ISSN 09658564. doi: 10.1016/j.tra.2018.01.017. URL https://linkinghub.elsevier.com/retrieve/ pii/s0965856417311771.

[7] Ehsan Shafiei, Brynhildur Davidsdottir, Hlynur Stefansson, Eyjolfur Ingi Asgeirsson, Reza Fazeli, Marías Halldór Gestsson, and Jonathan Leaver. Simulation-based appraisal of tax-induced electro-mobility promotion in Iceland and prospects for energy-economic development. 133:110894. ISSN 03014215. doi: 10.1016/j.enpol.2019.110894. URL https://linkinghub.elsevier.com/retrieve/pii/s0301421519304720.

[8] Modeste Kameni Nematchoua, José A. Orosa, and Sigrid Reiter. Energy consumption assessment due to the mobility of inhabitants and multiannual prospective on the horizon 2030-2050 in one Belgium city. 171:523-534. ISSN 03605442. doi: 10.1016/j.energy.2019.01.032. URL https://linkinghub.elsevier.com/retrieve/ pii/s0360544219300349.

[9] Neda Mohammadi and John E. Taylor. Urban energy flux: Spatiotemporal fluctuations of building energy consumption and human mobility-driven prediction. 195:810-818. ISSN 03062619. doi: 10.1016/j.apenergy.2017.03.044. URL https://linkinghub.elsevier.com/retrieve/pii/s0306261917302805.

[10] S. Bracco, C. Cancemi, F. Causa, M. Longo, and S. Siri. Optimization model for the design of a smart energy infrastructure with electric mobility. 51(9):200-205. ISSN 24058963. doi: 10.1016/j.ifacol.2018.07.033. URL https://linkinghub.elsevier.com/retrieve/pii/s2405896318307560. 
[11] Francesco Calise, Francesco Liberato Cappiello, Armando Cartenì, Massimo Dentice d'Accadia, and Maria Vicidomini. A novel paradigm for a sustainable mobility based on electric vehicles, photovoltaic panels and electric energy storage systems: Case studies for Naples and Salerno (Italy). 111:97-114. ISSN 13640321. doi: 10.1016/j.rser. 2019.05.022. URL https://linkinghub.elsevier.com/retrieve/pii/s1364032119303351.

[12] Katja Laurischkat and Daniel Jandt. Techno-economic analysis of sustainable mobility and energy solutions consisting of electric vehicles, photovoltaic systems and battery storages. 179:642-661. ISSN 09596526. doi: 10.1016/j.jclepro.2017.11.201. URL https://linkinghub.elsevier.com/retrieve/pii/ S0959652617328743.

[13] Matteo Muratori, Paige Jadun, Brian Bush, David Bielen, Laura Vimmerstedt, Jeff Gonder, Chris Gearhart, and Doug Arent. Future integrated mobility-energy systems: A modeling perspective. 119:109541. ISSN 13640321. doi: 10.1016/j.rser.2019.109541. URL https://linkinghub.elsevier.com/retrieve/ pii/S136403211930749X.

[14] Stefano Moret, Víctor Codina Gironès, Michel Bierlaire, and François Maréchal. Characterization of input uncertainties in strategic energy planning models. 202:597-617. ISSN 03062619. doi: 10.1016/j.apenergy.2017.05.106. URL https://linkinghub.elsevier.com/retrieve/pii/s0306261917306116.

[15] Stefano Moret. Strategic energy planning under uncertainty.

[16] Xiang Li, Theodoros Damartzis, Zoe Stadler, Stefano Moret, Boris Meier, Markus Friedl, and François Maréchal. Decarbonization in Complex Energy Systems: A Study on the Feasibility of Carbon Neutrality for Switzerland in 2050. 8. ISSN 2296-598X. doi: 10.3389/fenrg.2020.549615. URL https://www.frontiersin.org/ articles/10.3389/fenrg.2020.549615/full.

[17] RTE. Enjeux du développement de l'électromobilité pour le système Électrique, .

[18] BASt. BASt 2017 - Automatische Straßenverkehrszählung: Aktuelle Werte. URL https://www.bast.de/ BASt_2017/DE/Verkehrstechnik/Fachthemen/v2-verkehrszaehlung/Aktuell/zaehl_ aktuell_node.html.

[19] opendata_paris. Comptage routier - données trafic issues des capteurs permanents. URL https://opendata. paris.fr/explore/dataset/comptages-routiers-permanents/

[20] Martin Weiss, Andreas Zerfass, and Eckard Helmers. Fully electric and plug-in hybrid cars - An analysis of learning rates, user costs, and costs for mitigating CO2 and air pollutant emissions. 212:1478-1489. ISSN 0959-6526. doi: 10.1016/j.jclepro.2018.12.019. URL/http://www.sciencedirect.com/science/article/pii/ S0959652618337211.

[21] Eleonora Ruffini and Max Wei. Future costs of fuel cell electric vehicles in California using a learning rate approach. 150:329-341. ISSN 0360-5442. doi: 10.1016/j.energy.2018.02.071. URL http://www.sciencedirect. $\mathrm{com} / \mathrm{science/article/pii/S0360544218302998}$

[22] Fuquan Zhao, Kangda Chen, Han Hao, and Zongwei Liu. Challenges, Potential and Opportunities for Internal Combustion Engines in China. 12(12):4955. ISSN 2071-1050. doi: 10.3390/su12124955. URL https://www. mdpi.com/2071-1050/12/12/4955.

[23] Sylvain Moreau. Bilan énergétique de la france en 2018 - données provisoires. page 4.

[24] Arno Schroten, Lisanne van Wijngaarden, Marco Brambilla, Marco Gatto, Silvia Maffii, Frank Trosky, Kareen El Beyrouty, Sofia Amaral, Holger Krämer, Reinhard Monden, Damaris Bertschmann, Maura Killer, Vitalie Lambla, European Commission, Directorate General for Mobility and Transport, CE Delft, INFRAS, Ricardo, Te Rēhia Theatre, Planco, and ISL. Overview of transport infrastructure expenditures and costs. URL http:// publications.europa.eu/publication/manifestation_identifier/PUB_MI0518052ENN.

[25] RTE. Production - Hydraulique : RTE Bilan Électrique 2018, . URL https://bilan-electrique-2018. rte-france.com/hydraulique/\#.

[26] GSA. Morris Screening Method - gsa-module 0.5.3 documentation. URL https://gsa-module. readthedocs.io/en/stable/implementation/morris_screening_method.html

[27] Francesca Campolongo, Jessica Cariboni, and Andrea Saltelli. An effective screening design for sensitivity analysis of large models. 22(10):1509-1518. ISSN 1364-8152. doi: 10.1016/j.envsoft.2006.10.004. URL http://www . sciencedirect.com/science/article/pii/s1364815206002805. 